\title{
Análise dos pacientes internados em uma unidade de terapia intensiva com complicações por diabetes mellitus em Parnaíba-PI
}

\author{
Analysis of patients interned in na intensive care unit for complications of diabetes mellitus \\ in the municipality of Parnaíba-PI
}

\author{
Análisis de pacientes ingresados en una unidad de cuidados intensivos con \\ complicaciones por diabetes mellitus en Parnaíba-PI
}

Patrícia Sousa da Silveira ${ }^{1}$, Pedro Augusto Vieira Rosa Sousa ${ }^{1 *}$, Caliza Calmon de Araújo Mascarenhas ${ }^{1}$, Isabela Maria Rios Malta ${ }^{1}$, Rhuan Alves de Araújo ${ }^{1}$, Ananda Cordeiro Vieira ${ }^{2}$, Vanessa de Sousa do Vale ${ }^{3}$, Antonione Santos Bezerra Pinto ${ }^{1}$, Larissa Aguiar Luz Albuquerque ${ }^{1}$, Tereza Cristina de Carvalho Souza Garcês ${ }^{1}$.

\section{RESUMO}

Objetivo: Identificar o perfil epidemiológico e a evolução dos pacientes com complicações crônicas e agudas do diabetes, internados na Unidade de Terapia Intensiva (UTI) de um hospital público de referência na região litorânea do Piauí. Métodos: Trata-se de um estudo observacional, analítico, descritivo, retrospectivo, realizado entre de janeiro de 2016 e maio de 2019 através da análise de prontuários. Os dados foram organizados em planilhas do Microsoft Excel® e exportados para análise no software Statistical Package for the Social Sciences ${ }^{\circledR}$ (SPSS) versão 20.0. Resultados: O estudo analisou 84 prontuários, dentre as hipóteses diagnósticas mais reportadas no período, a infecção de ferimento e a descompensação aguda do diabetes obtiveram uma maior prevalência, ambas com $20,2 \%$ das internações. A amputação/desarticulação de membros inferiores foi registrada em $40,5 \%$ dos hospitalizados $(p=0,001)$, sendo o procedimento médico mais realizado nesse período. O óbito foi o principal motivo de saída, sendo registrada uma taxa de letalidade geral de $52,4 \%$ ( $p=0,199)$. Conclusão: As complicações diabéticas mais relatadas foram a descompensação aguda do diabetes e o ferimento infectado, com parte dos pacientes evoluindo para óbito durante o período. O presente estudo pode ser utilizado como base para o planejamento de medidas públicas de intervenção mais objetivas.

Palavras-chave: Diabetes mellitus, Saúde pública, Epidemiologia.

\begin{abstract}
Objective: To identify the epidemiological profile and the evolution of patients with chronic and acute complications of diabetes, admitted to the Intensive Care Unit (ICU) of a public reference hospital in the coastal region of Piauí. Methods: This is an observational, analytical, descriptive, retrospective study, carried out between January 2016 and May 2019 through the analysis of medical records. The data were organized in Microsoft Excel® spreadsheets and exported for analysis using the Statistical Package for the Social Sciences ${ }^{\circledR}$ (SPSS) version 20.0 software. Results: The study analyzed 84 medical records, among the most reported diagnostic hypotheses in the period, wound infection and acute decompensation of diabetes obtained a higher prevalence, both with $20.2 \%$ of hospitalizations. Amputation / disarticulation of lower limbs was recorded in $40.5 \%$ of hospitalized patients $(p=0.001)$, being the most common medical procedure during this period. Death was the most reported reason for leaving, with an overall lethality rate of $52.4 \%(p=0.199)$. Conclusion: The most reported diabetic complications were the acute decompensation of diabetes and the infected wound, with part of the patients progressing to death during the period. The present study can be used as a basis for planning more objective intervention measures.
\end{abstract}

Keywords: Diabetes mellitus, Public health, Epidemiology.

${ }_{1}^{1}$ Faculdade de Ciências Humanas, Exatas e da Saúde do Piauí/Instituto de Educação Superior do Vale do Parnaíba (FAHESP/IESVAP), Parnaíba - PI. *E-mail: rosapedro9@gmail.com

${ }^{2}$ Centro Universitário UNINOVAFAPI, Teresina - PI.

3 Universidade Federal do Maranhão (UFMA), São Bernardo - MA. 


\section{RESUMEN}

Objetivo: Identificar el perfil epidemiológico y la evolución de los pacientes con complicaciones crónicas y agudas de la diabetes ingresados en la Unidad de Cuidados Intensivos (UCl) de un hospital público de referencia de la región costera de Piauí. Métodos: Se trata de un estudio observacional, analítico, descriptivo, retrospectivo, realizado entre enero de 2016 y mayo de 2019 mediante el análisis de historias clínicas. Los datos se organizaron en hojas de cálculo de Microsoft Excel® y se exportaron para su análisis utilizando el software Statistical Package for the Social Sciences ${ }^{\circledR}$ (SPSS) versión 20.0. Resultados: El estudio analizó 84 historias clínicas, entre las hipótesis diagnósticas más reportadas en el período, la infección de la herida y la descompensación aguda de la diabetes obtuvieron una mayor prevalencia, ambas con el $20,2 \%$ de las hospitalizaciones. La amputación / desarticulación de miembros inferiores se registró en el 40,5\% de los hospitalizados $(p=0,001)$, siendo el procedimiento médico más común durante este período. La muerte fue la razón más reportada para irse, con una tasa de letalidad general del 52,4\% $(p=0,199)$. Conclusión: Las complicaciones diabéticas más reportadas fueron la descompensación aguda de la diabetes y la herida infectada, con una parte de los pacientes que progresaron a la muerte durante el período. El presente estudio puede utilizarse como base para planificar medidas de intervención más objetivas.

Palabras clave: Diabetes mellitus, Salud pública, Epidemiología.

\section{INTRODUÇÃO}

Tendo em vista o atual cenário do século XXI, nota-se um crescente aumento de pacientes com doenças crônicas, entre elas o diabetes mellitus (DM) que constitui um problema de saúde pública mundial (COCKERHAM WC, et al., 2017; ZHANG P, et al., 2016). O sedentarismo e a dieta rica em gorduras associado aos elevados índices de inatividade física, são hábitos de vida intensamente difundidos no contexto atual e preconizados por uma numerosa parte da população, contribuindo para que o diabetes se tornasse uma epidemia de proporções globais (COSTA AF, et al., 2017). São 463 milhões de adultos diabéticos em todo o mundo e os países de economias emergentes possuem os maiores índices, entre eles o Brasil que ocupa o 4 lugar no ranking dos países com maior prevalência de indivíduos diabéticos (INTERNATIONAL DIABETES FEDERATION, 2019).

O DM consiste em um distúrbio metabólico caracterizado por hiperglicemia persistente, decorrente da deficiência na produção de insulina ou na sua ação, ou em ambos os mecanismos (SOCIEDADE BRASILEIRA DE DIABETES, 2019). O mau controle glicêmico por um período prolongado de tempo pode ocasionar o surgimento de complicações associadas ao DM que podem ser divididas em agudas e crônicas (WU T, et al., 2017). Dentre as complicações agudas destacam-se a hipoglicemia, a cetoacidose diabética e a síndrome hiperglicêmica hiperosmolar não-cetônica (GINTER E e SIMKO V, 2012; AMERICAN DIABETES ASSOCIATION, 2013).

As crônicas por sua vez englobam complicações macrovasculares e microvasculares, de acordo com o conjunto de vasos sanguíneos afetados pela hiperglicemia. As microvasculares são caracterizadas pela retinopatia diabética, doença renal do diabetes e neuropatia diabética, que constituem as causas mais comuns de cegueira irreversível, doença renal crônica e amputações não traumáticas de membros inferiores (QUEIROZ PC, et al., 2011; SANTOS JC e MOREIRA TMM, 2012). Em nível macrovascular, destacam-se a cardiopatia isquêmica, a doença cerebrovascular e a doença vascular periférica, que estão frequentemente associadas à morbimortalidade decorrente da doença (CENCI DR, et al., 2013).

Além disso, de acordo com o atlas da International Diabetes Federation (IDF) de 2017 a Doença Cardiovascular (DCV) é a principal causa de morte e incapacidade nos casos de diabetes, sendo que $44 \%$ das pessoas com DM desenvolvem doença renal crônica e a prevalência de neuropatia periférica varia de $16 \%$ a $66 \%$. As complicações relacionadas ao DM são preponderantes no aumento da mortalidade, causando aproximadamente dois milhões de mortes a cada ano em todo o mundo (NCD RISK FACTOR COLLABORATION, 2016).

Esse cenário tem gerado altos custos sociais e financeiros, tanto aos pacientes quanto ao sistema público de saúde. Estima-se que em 2013 as internações decorrentes de condições diabéticas custaram cerca de R\$ 
463 milhões, representando cerca de $4,3 \%$ do custo total de hospitalizações no Sistema Único de Saúde (SUS), tendo em vista que a cronicidade da doença demanda um alto número de procedimentos diagnósticos e terapêuticos, além de comumente ocasionar estados de invalidez e morte prematura entre a população (BRASIL, 2013).

As investigações epidemiológicas contidas no presente estudo podem não só guiar medidas de prevenção com foco na complicação mais prevalente, como também auxiliar no aumento da eficácia das medidas de tratamento voltadas aos indivíduos diabéticos. Dessa forma, a partir da definição das características sociodemográficas e patológicas mais comuns, os sistemas públicos de saúde podem direcionar e intensificar as ações de cuidado que serão dirigidas a essa parcela específica da população (GIMENES C, et al., 2016).

Dessa forma, levando-se em consideração a relevância do DM, bem como o seu grande número de complicações, o presente estudo buscou investigar quais os tipos de complicações agudas e crônicas tratadas na instituição e que acometem em maior número os portadores do diabetes, além de acompanhar a evolução dos casos de DM em um hospital público de referência na região, com a finalidade de traçar o perfil epidemiológico mais prevalente para essa condição.

\section{MÉTODOS}

Trata-se de um estudo observacional, descritivo, retrospectivo, no qual foi realizada uma análise sobre os casos de complicações do DM admitidos na Unidade de Terapia Intensiva (UTI) de um hospital de referência da planície litorânea situado em Parnaíba, no período de janeiro de 2016 a maio de 2019. A cidade está localizada na região norte do Piauí e a instituição hospitalar supracitada é referência para onze municípios vizinhos que compõem a planície litorânea piauiense, além de receber eventualmente, pacientes procedentes dos estados do Maranhão e Ceará.

Os dados foram obtidos através da análise de prontuários, sendo selecionados todos os que continham informações como idade, sexo, procedência, procedimento solicitado, hipótese diagnóstica a partir da evolução do paciente na UTI, período de internação, motivo de saída e comorbidades. Essas variáveis foram organizadas em qualitativas e quantitativas para análise dos resultados.

As informações coletadas foram registradas em um banco de dados na Planilha do Programa Microsoft Excel ${ }^{\circledR}$ e exportadas para análises no software Statistical Package for the Social Sciences ${ }^{\circledR}$ (SPSS) versão 20. Os resultados foram descritos em tabelas de frequência absoluta e percentual. As associações/relações entre as variáveis foram obtidas pelo teste Qui-quadrado, sendo que o nível de significância estabelecido foi $5 \%(p<0.05)$ e o nível de confiança adotado foi de $95 \%$.

A pesquisa foi realizada conforme as Diretrizes e Normas Regulamentadoras de Pesquisa Envolvendo Seres Humanos, seguindo as Resoluções no 466/2012 e no 510/2016 do Conselho Nacional de Saúde. O estudo foi aprovado pelo Comitê de Ética em Pesquisa do Centro Universitário Tocantinense Presidente Antônio Carlos (UNITPAC), sob aprovação do CAAE: 17711019.0.0000.0014, bem como pelo setor responsável da instituição hospitalar na qual o estudo foi realizado.

\section{RESULTADOS}

Durante o período de janeiro de 2016 e maio de 2019, foram registradas 84 internações relacionadas a complicações do diabetes mellitus, sendo que 19,04\% ocorreram em 2016, 30,95\% em 2017, 41,67\% em 2018 e 19,04\% das hospitalizações ocorreram nos cinco primeiros meses do ano de 2019.

A média de idade dos pacientes internados durante o período foi de 65,3 anos, com $60,7 \%$ das internações correspondendo a indivíduos com idade superior a 65 anos e 2,4\% a menores de 25 anos. Durante o estudo, foi identificada uma maior prevalência de pessoas do sexo masculino, com $57,1 \%$ dos casos. Em relação à procedência desses pacientes, foi registrado que $48,8 \%$ são de Parnaíba- $\mathrm{PI}$, seguido de Luís Correia-PI com $7,1 \%$. Entretanto, observou-se que o hospital recebe pacientes procedentes de outras cidades do Piauí e também de municípios dos estados vizinhos do Maranhão e Ceará (Tabela 1). 
Tabela 1 - Casos analisados de acordo com a faixa etária, sexo e procedência.

\begin{tabular}{ccc}
\hline Variáveis & $\mathbf{n}^{*}$ & $\%$ \\
\hline Faixa etária & 2 & 2,4 \\
\hline$<25$ & 6 & 7,1 \\
$25-39$ & 11 & 13,1 \\
$40-54$ & 14 & 16,7 \\
$55-64$ & 51 & 60,7 \\
$>=65$ & & \\
\hline Sexo & 36 & 42,9 \\
\hline Feminino & 48 & 57,1 \\
Masculino & & \\
\hline Procedência & & 48,8 \\
\hline Piauí & 41 & 7,1 \\
\hline Parnaíba - PI & 6 & 3,6 \\
Luís Correia - PI & 3 & 22,6 \\
Buriti dos Lopes-PI & 19 & \\
Outros - PI & & 6,0 \\
\hline Maranhão & 5 & 3,6 \\
\hline Tutóia - MA & 3 & 2,4 \\
São Bernardo - MA & 2 & 4,8 \\
Santana - MA & 4 & \\
Outros - MA & & \\
\hline Ceará & 1 & \\
\hline Barroquinha-CE & & \\
\hline
\end{tabular}

Legenda: *n: Número amostral

Fonte: Silveira PS, et al., 2021.

De acordo com as informações contidas nos prontuários, $40,5 \%$ dos pacientes $(p=0,001)$ foram admitidos na Unidade de Terapia Intensiva (UTI) com o procedimento solicitado de amputação/desarticulação de membros inferiores (MMII), sendo que esse procedimento foi o mais realizado no ano de 2018, seguido por tratamento de descompensação aguda do DM (DADM) com 22,6\% ( $p=0,001)$ dos casos, o qual teve maior prevalência no ano de 2016 (Tabela 2). Outros procedimentos como o tratamento de complicações macrovasculares do DM como a doença vascular periférica, a insuficiência renal (IR) e o acidente vascular encefálico (AVE) também foram relatados, porém em menor quantidade.

Tabela 2 - Descrição dos procedimentos solicitados na admissão na UTI durante o período analisado.

\begin{tabular}{|c|c|c|c|c|c|c|c|c|c|c|c|}
\hline \multirow{3}{*}{ Variáveis } & \multicolumn{6}{|c|}{ Ano } & & & \multirow{2}{*}{\multicolumn{2}{|c|}{ Total }} & \multirow{3}{*}{$\begin{array}{c}\text { p- } \\
\text { valor }\end{array}$} \\
\hline & \multicolumn{2}{|c|}{2016} & \multicolumn{2}{|c|}{2017} & \multicolumn{2}{|c|}{2018} & \multicolumn{2}{|c|}{2019} & & & \\
\hline & $\mathrm{n}^{\dagger}$ & $\%$ & $\mathbf{N}$ & $\%$ & $\mathbf{N}$ & $\%$ & $\mathbf{N}$ & $\%$ & $\mathbf{n}$ & $\%$ & \\
\hline Procedimento solicitado & & & & & & & & & & & 0,001 \\
\hline Amputação/Desarticulação de MMII & 2 & 2,4 & 12 & 14,3 & 18 & 21,4 & 2 & 2,4 & 34 & 40,5 & \\
\hline Tratamento de AVE & 2 & 2,4 & 0 & 0,0 & 0 & 0,0 & 0 & 0,0 & 2 & 2,4 & \\
\hline $\begin{array}{l}\text { Tratamento de complicações } \\
\text { macrovasculares do DM }\end{array}$ & 1 & 1,2 & 5 & 6,0 & 4 & 4,8 & 1 & 1,2 & 11 & 13,1 & \\
\hline Tratamento por DADM & 10 & 11,9 & 4 & 4,8 & 3 & 3,6 & 2 & 2,4 & 19 & 22,6 & \\
\hline Tratamento de IR & 1 & 1,2 & 5 & 6,0 & 10 & 11,9 & 2 & 2,4 & 18 & 21,4 & \\
\hline
\end{tabular}

Legenda: ${ }^{\star}$ Teste qui-quadrado de Pearson; ${ }^{n} \mathrm{n}$ : Número amostral; MMII: Membros inferiores, AVE: Acidente Vascular Encefálico, DM: Diabetes mellitus; DADM: Descompensação aguda do diabetes mellitus, IR: Insuficiência renal.

Fonte: Silveira PS, et al., 2021. 
Ao analisar os prontuários, foram coletadas informações relacionadas à hipótese diagnóstica, que foi formulada de acordo com a evolução do paciente a partir do momento da admissão na instituição, sendo que as mais encontradas foram: ferimento infectado com $20,2 \%$, descompensação aguda do diabetes com $20,2 \%$ e Amputação de Membro Inferior (MMII) com 16,7\% dos casos. Outras hipóteses, assim como a presença da correlação entre elas, também foram registradas (Tabela 3).

Tabela 3 - Hipótese diagnóstica a partir da evolução do paciente na UTI* e suas correlações em pacientes internados por complicações diabéticas no Hospital de referência da planície litorânea.

\begin{tabular}{ccc}
\hline Hipótese diagnóstica & $\mathbf{n}^{\dagger}$ & $\%$ \\
\hline Acidente vascular encefálico & 2 & 2,4 \\
Amputação de MMII & 14 & 16,7 \\
Amputação de MMII + Descompensação aguda do diabetes & 1 & 1,2 \\
Descompensação aguda do diabetes & 17 & 20,2 \\
Ferimento infectado & 17 & 20,2 \\
Insuficiência renal & 13 & 15,5 \\
Insuficiência renal + Descompensação aguda do diabetes & 1 & 1,2 \\
Insuficiência renal + Ferimento infectado & 2 & 2,4 \\
Doença renal do diabetes & 3 & 3,6 \\
Pé diabético & 9 & 10,7 \\
Sepse em membros inferiores & 3 & 3,6 \\
Outros & 2 & 2,3 \\
\hline
\end{tabular}

Legenda: *UTI: Unidade de Terapia Intensiva; + n: Número amostral; MMII: Membros inferiores

Fonte: Silveira PS, et al., 2021.

De acordo com a evolução dos pacientes, a média do período de internação foi de 21,2 dias, com a amplitude variando de 1 até 204 dias de hospitalização (desvio padrão=23,9). Dentre os motivos para saída da UTI, 52,4\% corresponderam a evoluções para óbito $(p=0,199)$, caracterizando a taxa de letalidade geral. Além disso, $29,8 \%$ desses pacientes eram pertencentes ao sexo feminino e $22,6 \%$ ao sexo masculino ( $p=$ $0,031)$. A alta hospitalar apareceu em seguida, com $45,2 \%(p=0,018)$. Os demais pacientes foram reoperados ou transferidos (Tabela 4).

Tabela 4 - Distribuição dos casos quanto ao sexo relacionados com o motivo da saída.

\begin{tabular}{|c|c|c|c|c|c|c|c|}
\hline \multirow{3}{*}{ Variáveis } & \multicolumn{4}{|c|}{ Sexo } & \multirow{2}{*}{\multicolumn{2}{|c|}{ Total }} & \multirow{3}{*}{ p-valor } \\
\hline & \multicolumn{2}{|c|}{ Feminino } & \multicolumn{2}{|c|}{ Masculino } & & & \\
\hline & $\mathbf{n}^{\dagger}$ & $\%$ & $\mathbf{n}$ & $\%$ & $\mathbf{N}$ & $\%$ & \\
\hline Motivo da saída & & & & & & & $0,018^{\star}$ \\
\hline Alta & 11 & 13,1 & 27 & 32,1 & 38 & 45,2 & \\
\hline Óbito & 25 & 29,8 & 19 & 22,6 & 44 & 52,4 & \\
\hline Reoperação/Transf. & 0 & 0,0 & 2 & 2,4 & 2 & 2,4 & \\
\hline
\end{tabular}

Legenda: *Teste qui-quadrado de Pearson; $†$ n: Número amostral

Fonte: Silveira PS, et al., 2021.

A partir da análise estatística, notou-se que a principal hipótese diagnóstica relacionada com a maior taxa de óbito foi a de ferimento infectado, pois $11,9 \%$ dos pacientes $(p<0,001)$ que possuíam características clínicas que levaram à definição dessa hipótese evoluíram ao óbito. Os hospitalizados com hipóteses diagnósticas de IR e DADM, obtiveram taxas de letalidade idênticas, ocorrendo morte em $9,5 \%$ dos casos $(p<0,001)$ (Tabela 5). 
Tabela 5 - Distribuição do motivo de saída relacionado com a hipótese diagnóstica.

\begin{tabular}{|c|c|c|c|c|c|c|c|c|c|}
\hline \multirow{3}{*}{ Variáveis } & \multicolumn{6}{|c|}{ Motivo da saída } & \multirow{2}{*}{\multicolumn{2}{|c|}{ Total }} & \multirow{3}{*}{$\begin{array}{l}\text { p-valor } \\
<0,001\end{array}$} \\
\hline & \multicolumn{2}{|c|}{ Alta } & \multicolumn{2}{|c|}{ Óbito } & \multicolumn{2}{|c|}{$\begin{array}{c}\text { Reopera- } \\
\text { ção/Transf. }\end{array}$} & & & \\
\hline & $\mathbf{n}^{\dagger}$ & $\%$ & $\mathbf{n}$ & $\%$ & $\mathbf{n}$ & $\%$ & $\mathbf{n}$ & $\%$ & \\
\hline Acidente vascular encefálico & 0 & 0,0 & 2 & 2,4 & 0 & 0,0 & 2 & 2,4 & \\
\hline Amputação de MMII & 7 & 8,3 & 7 & 8,3 & 0 & 0,0 & 14 & 16,7 & \\
\hline Amputação de MMII + DADM & 0 & 0,0 & 0 & 0,0 & 1 & 1,2 & 1 & 1,2 & \\
\hline DADM & 9 & 10,7 & 8 & 9,5 & 0 & 0,0 & 17 & 20,2 & \\
\hline Ferimento infectado & 7 & 8,3 & 10 & 11,9 & 0 & 0,0 & 17 & 20,2 & \\
\hline IR & 5 & 6,0 & 8 & 9,5 & 0 & 0,0 & 13 & 15,5 & \\
\hline IR + DADM & 1 & 1,2 & 0 & 0,0 & 0 & 0,0 & 1 & 1,2 & \\
\hline IR + ferimento infectado & 2 & 2,4 & 0 & 0,0 & 0 & 0,0 & 2 & 2,4 & \\
\hline Doença renal do diabetes & 1 & 1,2 & 2 & 2,4 & 0 & 0,0 & 3 & 3,6 & \\
\hline Pé diabético & 5 & 6,0 & 3 & 3,6 & 1 & 1,2 & 9 & 10,7 & \\
\hline Sepse em MMII & 0 & 0,0 & 3 & 3,6 & 0 & 0,0 & 3 & 3,6 & \\
\hline Outros & 1 & 1,2 & 1 & 1,2 & 0 & 0,0 & 2 & 2,4 & \\
\hline
\end{tabular}

Legenda: *Teste qui-quadrado de Pearson. ${ }^{\dagger} \mathrm{n}$ : Número amostral; MMII: Membros inferiores, DADM: Descompensação aguda do diabetes mellitus, IR: insuficiência renal.

Fonte: Silveira PS, et al., 2021.

Além disso, os pacientes apresentavam algumas comorbidades, entre elas a Hipertensão arterial (HAS) que foi registrada em $56 \%$ dos pacientes $(p=0,272)$, sendo mais prevalente em maiores de 65 anos de idade, seguida pela IR em $11,9 \%$ dos casos $(p=0,002)$. Outras comorbidades também foram descritas, porém em menores quantidades (Tabela 6).

Tabela 6 - Distribuição das faixas etárias relacionadas com sexo e as comorbidade.

\begin{tabular}{|c|c|c|c|c|c|c|c|c|c|c|c|c|c|}
\hline \multirow{3}{*}{ Variáveis } & \multicolumn{10}{|c|}{ Faixa etária } & \multirow{2}{*}{\multicolumn{2}{|c|}{ Total }} & \multirow{3}{*}{ p-valor* } \\
\hline & \multicolumn{2}{|c|}{$<25$} & \multicolumn{2}{|c|}{$25-39$} & \multicolumn{2}{|c|}{$40-54$} & \multicolumn{2}{|c|}{$55-64$} & \multicolumn{2}{|c|}{$>=65$} & & & \\
\hline & $\mathbf{n}^{\dagger}$ & $\%$ & $\mathbf{n}$ & $\%$ & $n$ & $\%$ & $\mathbf{n}$ & $\%$ & $\mathbf{n}$ & $\%$ & $\mathbf{n}$ & $\%$ & \\
\hline Sexo & & & & & & & & & & & & & 0,031 \\
\hline Feminino & 2 & 2,4 & 2 & 2,4 & 1 & 1,2 & 9 & 10,7 & 22 & 26,2 & 36 & 42,9 & \\
\hline Masculino & 0 & 0,0 & 4 & 4,8 & 10 & 11,9 & 5 & 6,0 & 29 & 34,5 & 48 & 57,1 & \\
\hline HAS & & & & & & & & & & & & & 0,272 \\
\hline Sim & 0 & 0,0 & 2 & 2,4 & 5 & 6,0 & 9 & 10,7 & 31 & 36,9 & 47 & 56,0 & \\
\hline Não & 2 & 2,4 & 4 & 4,8 & 6 & 7,1 & 5 & 6,0 & 20 & 23,8 & 37 & 44,0 & \\
\hline IR & & & & & & & & & & & & & 0,002 \\
\hline Sim & 0 & 0,0 & 1 & 1,2 & 0 & 0,0 & 6 & 7,1 & 3 & 3,6 & 10 & 11,9 & \\
\hline Não & 2 & 2,4 & 5 & 6,0 & 11 & 13,1 & 8 & 9,5 & 48 & 57,1 & 74 & 88,1 & \\
\hline Nefropatia & & & & & & & & & & & & & 0,002 \\
\hline Sim & 1 & 1,2 & 2 & 2,4 & 0 & 0,0 & 0 & 0,0 & 2 & 2,4 & 5 & 6,0 & \\
\hline Não & 1 & 1,2 & 4 & 4,8 & 11 & 13,1 & 14 & 16,7 & 49 & 58,3 & 79 & 94,0 & \\
\hline AVE & & & & & & & & & & & & & 0,382 \\
\hline Sim & 0 & 0,0 & 0 & 0,0 & 0 & 0,0 & 0 & 0,0 & 6 & 7,1 & 6 & 7,1 & \\
\hline Não & 2 & 2,4 & 6 & 7,1 & 11 & 13,1 & 14 & 16,7 & 45 & 53,6 & 78 & 92,9 & \\
\hline Pneumonia & & & & & & & & & & & & & 0,028 \\
\hline Sim & 1 & 1,2 & 0 & 0,0 & 1 & 1,2 & 1 & 1,2 & 1 & 1,2 & 4 & 4,8 & \\
\hline Não & 1 & 1,2 & 6 & 7,1 & 10 & 11,9 & 13 & 15,5 & 50 & 59,5 & 80 & 95,2 & \\
\hline Outros & & & & & & & & & & & & & 0,047 \\
\hline Sim & 0 & 0,0 & 2 & 2,4 & 6 & 7,1 & 2 & 2,4 & 8 & 9,5 & 18 & 21,4 & \\
\hline Não & 2 & 2,4 & 4 & 4,8 & 5 & 6,0 & 12 & 14,3 & 43 & 51,2 & 66 & 78,6 & \\
\hline
\end{tabular}

Legenda: *Teste qui-quadrado de Pearson. ${ }^{\dagger}$ n: Número amostral; HAS: Hipertensão arterial sistólica, IR: Insuficiência renal, AVE: Acidente vascular encefálico.

Fonte: Silveira PS, et al., 2021. 


\section{DISCUSSÃO}

O presente estudo avaliou os casos de internação decorrentes de descompensações do diabetes mellitus, e partir da análise dos prontuários, observou-se que as complicações diabéticas ainda possuem uma notável prevalência na área da planície litorânea do Piauí, sendo preponderantes para um número considerável de hospitalizações na instituição de saúde de referência da região supracitada.

A fonte de dados permitiu acesso às variáveis qualitativas e quantitativas, como também a informações acerca dos procedimentos técnicos documentados realizados durante o período de internação de cada paciente, permitindo ao estudo confeccionar um panorama epidemiológico relacionado à temática, bem como acompanhar a evolução dos pacientes. Durante a pesquisa foram avaliadas complicações crônicas como acidente vascular encefálico, doença vascular periférica, ferimento infectado, doença renal do diabetes e o pé diabético, além de complicações decorrentes dos estados de descompensação aguda do diabetes, conforme os dados descritos nos prontuários dos pacientes.

O perfil sociodemográfico obtido pelo presente estudo mostrou que $57,1 \%$ das hospitalizações foram atribuídas a indivíduos do sexo masculino e que apesar do hospital receber demandas oriundas de outros municípios, grande parte dos pacientes atendidos eram procedentes da cidade Parnaíba-PI, na qual o hospital está localizado. Resultado similar foi encontrado pelos autores Tang ZQ, et al. (2014), Bertolini DNP e Simonetti JP (2014), os quais apontaram a falta de autocuidado alimentar e negligência quanto às orientações terapêuticas, como particularidades mais comuns a indivíduos do sexo masculino.

As internações analisadas no período acometeram principalmente idosos, haja vista que a média final de idade entre os indivíduos hospitalizados durante o período foi de 65,3 anos, com $60,7 \%$ das internações correspondendo a pacientes com idade superior a 65 anos de idade. Essas informações estão de acordo com os achados de diversos outros trabalhos disponíveis na literatura científica mundial, como os estudos de Cecílio HP, et al. (2015), Santos AL, et al. (2015) e Prado MAMB, et al. (2016), que confirmaram a maior prevalência de acometimento de pessoas de idade avançada por doenças crônicas não transmissíveis.

A constatação da existência de uma maior taxa de internação envolvendo pacientes idosos instiga enorme preocupação, uma vez que indivíduos diabéticos pertencentes a essa faixa etária são afetados de forma mais grave pelos diversos efeitos deletérios corporais inerentes a essa afecção, não somente com a injúria renal e as lesões cardiovasculares, como também por meio das alterações patológicas que predispõem a um maior risco de perda da acuidade visual e amputação de membros, o que aumenta consideravelmente a taxa de óbitos em pacientes com idade avançada (STOPA SR, et al., 2014; PIMENTA FB, et al., 2015).

Dentre os objetivos da pesquisa, a análise das comorbidades presentes foi de grande importância na associação com o prognóstico dos pacientes internados. As condições patológicas concomitantes contribuem determinantemente para a situação de desfecho da hospitalização por afecções diabéticas, pois elas maximizam os danos causados pelos eventos fisiopatológicos do diabetes, intensificando as lesões em múltiplos órgãos alvo (SCHMIDT MI, et al., 2011; CORTEZ DN, et al., 2015; GARCIA C, et al., 2016; WEXLER DJ, et al., 2020).

No presente estudo, a Hipertensão Arterial Sistólica (HAS) foi a principal comorbidade associada, com $56 \%$ dos pacientes diabéticos, proporção semelhante foi encontrada na análise transversal de Artilheiro MMVSA, et al. (2014), realizada na cidade de Joinville (SC), na qual foi detectado que $52 \%$ dos portadores de diabetes, usuários dos sistemas de saúde daquele município, eram também acometidos por HAS no momento da hospitalização.

Portanto, tendo em vista o curso altamente evitável dessa patologia e a partir da análise dos índices de comorbidades, é necessário que haja o fortalecimento das ações nos programas de atenção, rastreio e mudança de hábitos de vida das Unidades de Sáude Básica (UBS), visando à promoção de saúde e adoção de medidas de autocuidado entre a população. A atuação dessas instituições deve ser baseada nos dados obtidos pelo Sistema de Cadastramento e Acompanhamento de Hipertensos e Diabéticos (HIPERDIA), do Governo Federal. 
O HIPERDIA foi idealizado para auxiliar na orientação de pacientes da atenção primária e também fornecer assistência farmacêutica no suprimento contínuo de medicamentos, bem como no monitoramento das condições clínicas dos usuários dos serviços de saúde, sendo essencial no controle de agravos relacionados às complicações do estado diabético, uma vez que esse sistema de dados permite que os profissionais de saúde realizem um acompanhamento evolutivo das condições crônicas supracitadas (SANTOS AL, et al., 2018).

Com a análise das informações clínicas contidas nos prontuários analisados, evidenciou-se uma grande quantidade de casos de repercussões macrovasculares do diabetes, que possuem grande influência no aumento dos gastos hospitalares e de previdência social às instituições públicas do país (JÚNIOR EVS, et al., 2019). Os ferimentos infectados em membros inferiores foram responsáveis por numerosos casos de amputação, correspondendo a $40,5 \%$ dos procedimentos realizados em pacientes hospitalizados por complicações diabéticas durante o período, resultado similar foi identificado no estudo epidemiológico de Santos ICRV, et al. (2013), realizado na cidade de Recife (PE), que encontrou uma taxa de 50\% de desarticulações nas internações de pacientes portadores de pé diabético.

O estudo realizado por Marques ADB, et al. (2018), também obteve resultado semelhante, o qual associou o elevado número de casos de ferimentos infectados de membros inferiores à autonegligência corporal dos pacientes portadores do diabetes. O estresse local excessivo em membros, o descontrole glicêmico, o tabagismo e a demora na procura de atendimento hospitalar, observado em pacientes negligentes, são preponderantes para o aumento da gravidade dos ferimentos infectados (WEINTROB AC, et al., 2018; ARMSTRONG AG, et al., 2019). Tendo em vista que essa complicação é altamente evitável desde que as medidas de precaução e tratamento sejam tomadas em estágios precoces (CUBAS MR, et al., 2013).

Assim, se faz cada vez mais necessário que os sistemas públicos de saúde estejam aptos a atuarem na intervenção do problema, com a promoção de medidas de educação em saúde destinadas a esse público alvo, bem como na difusão de técnicas de cuidado pessoal e conscientização desses indivíduos acerca da importância de procurar assistência hospitalar imediata caso haja o acometimento por lesões desse tipo (MENEZES LCG DE, et al.,2017).

Outra importante complicação relacionada ao diabetes mellitus, foi descrita como "Cetoacidose diabética" (CAD) nos prontuários médicos e que, juntamente com o ferimento infectado, constituíram-se nas mais prevalentes do estudo. Entretanto, no decorrer da pesquisa foi adotada a nomenclatura "descompensação aguda do diabetes" como padronização desse diagnóstico, visto que, em alguns prontuários, não se observou o registro de exames laboratoriais essenciais para a confirmação desta condição, bem como para diagnóstico diferencial com Estado Hiperosmolar Hiperglicêmico não Cetótico (EHHNC).

A descompensação aguda do diabetes constituiu-se como a principal causa de óbitos em crianças, adolescentes e jovens portadores de DM. Taxas de mortalidade similares foram encontradas também por Levitsky LL, et al. (2019) e Glaser M, et al. (2019), estudos nos quais houve a correlação entre a alta mortalidade de indivíduos dessa faixa etária, e a semelhança sintomatológica entre os quadros de descompensação e outras patologias comuns na idade juvenil, como por exemplo, doenças que cursam com vômitos e desidratação.

Assim, a inespecificidade sintomática pode dificultar o diagnóstico correto e atrasar o início do tratamento. Entretanto, mesmo com as consideráveis taxas de mortalidade, a CAD e o EHHNC ainda possuem um índice diagnóstico insatisfatório, sobretudo nos serviços públicos de saúde, com apenas $42,3 \%$ dos casos sendo diagnosticados e tratados de forma correta (GOMES MB, et al., 2012).

Além disso, é importante enfatizar o índice de mortalidade durante o período analisado. O óbito foi o principal desfecho, sendo registrado em $52,4 \%$ dos casos, apresentando tendências semelhantes evidenciadas pelo estudo Global Burden of Disease, realizado em 195 países, o qual mostrou um aumento da mortalidade por DM no Brasil, nos anos de 1990 a 2015, de 35,9/100 mil em 1990 para 37,5/100 mil em 2015 (DUNCAN BB, et al., 2017). 
O desfecho de internação analisado na pesquisa é resultado da associação de medidas de autocuidado pessoal com a atuação dos mais diversos níveis de atenção à saúde pública. Dessa forma, os efeitos deletérios da hiperglicemia que persistem por um longo período de tempo e são comuns em pacientes negligentes quanto ao próprio cuidado corporal, além da inadequada implantação de estratégias de promoção e prevenção de saúde nos mais diversos níveis de complexidade de atendimentos aos pacientes diabéticos, contribuem decisivamente para a piora do prognóstico dessa patologia no país (GARCES TS, et al., 2018).

Dessa forma, o presente estudo e as informações epidemiológicas nele contidas, podem auxiliar no aumento da eficácia das estratégias públicas de saúde na Atenção Primária, como o programa HIPERDIA. O uso e associação dessas ferramentas também podem propiciar uma melhora da qualidade de rastreio das complicações diabéticas, utilizando-se dos fatores de risco mais prevalentes a essas repercussões patológicas para promover a redução das taxas de incidência, mortalidade e sequelas inerentes ao curso das descompensações decorrentes do diabetes mellitus. Além disso, o aperfeiçoamento na abordagem das condições diabéticas descompensadas pode proporcionar um melhor treinamento e capacitação em técnicas de saúde, para os profissionais que participarão do cuidado hospitalar disponibilizado a esses pacientes.

\section{CONCLUSÃO}

O perfil epidemiológico predominante constituiu-se em indivíduos do sexo masculino, idade superior a 65 anos e com HAS. As maiores causas de internação decorreram de complicações agudas do diabetes e por ferimentos infectados, demandando alto número de amputações e desarticulações. Ademais, $52,4 \%$ dos pacientes, evoluíram a óbito. Com isso, as intervenções voltadas para a prevenção de infecções hospitalares e o aperfeiçoamento dos protocolos clínicos são essenciais na redução dos índices de mortalidade desses pacientes. Assim, os resultados obtidos podem ser úteis no planejamento dessas ações, visto que a definição dos perfis sociodemográficos mais prevalentes permitem que os sistemas governamentais de saúde atuem de forma mais objetiva na implementação de projetos para a resolução dessa problemática.

\section{REFERÊNCIAS}

1. ADA. American Diabetes Association. Standards of Medical Care in Diabetes. Diabetes Care. 2013. Disponível em: https://care.diabetesjournals.org/content/44/Supplement_1. Acesso em: 12 de março de 2021.

2. ARMSTRONG DG, et al. Management of diabetic foot ulcers. UpToDate. 2019. Disponivel em:< https://www.uptodate.com/contents/management-of-diabetic-foot-ulcers>. Acesso em: 2 de janeiro de 2020.

3. ARTILHEIRO MMVSA, et al. Quem são e como são tratados os pacientes que internam por diabetes mellitus no SUS ?. Revista Saúde Debate, 2014; 8: 210-224.

4. BERTOLINI DNP, SIMONETTI JP. O gênero masculino e os cuidados de saúde: A experiência de homens de um centro de saúde. Escola Anna Nery revista de enfermagem, 2014; 18(4):722-727

5. BRASIL. Diretrizes da Sociedade Brasileira de Diabetes 2019-2020. Disponível em: https://www.diabetes.org.br/profissionais/images/DIRETRIZES-COMPLETA-2019-2020.pdf. Acesso em: 13 de fevereiro de 2020.

6. BRASIL. Sistemas de Informações Hospitalares- DATASUS. Disponível em: http://www2.datasus.gov.br/. Acesso em: 04 de abril de 2020.

7. CECILIO HP, et al. Comportamentos e comorbidades associados às complicações microvasculares do diabetes. Acta Paul Enferm, 2015; 28(2):113-9

8. CENCI DR, et al. Análise do equilíbrio em pacientes diabéticos por meio do sistema F-Scan e da Escala de Equilíbrio de Berg. Fisiot Mov, 2013; 26(1):55-61.

9. COCKERHAM WC, et al. The Social Determinants of Chronic Disease. Birmingham, Alabama: Division of Preventive Medicine, Department of Medicine, University of Alabama at Birmingham, 2017.

10. CORTEZ DN, et al. Complicações e o tempo de diagnóstico do diabetes mellitus na atenção primária. Escola Paulista de Enfermagem, 2015; 28(3): 250-5.

11. COSTA AF, et al. Carga do diabetes mellitus tipo 2 no Brasil. Cad. Saúde Pública, 2017; 33(2): e00197915.

12. CUBAS MR, et al. Pé diabético: orientações e conhecimento sobre cuidados preventivos. Fisioterapia em Movimento, 2013; 26(3): 647-55

13. DUNCAN BB, et al. The burden of diabetes and hyperglycemia in Brazil-past and present: findings from the Global Burden of Disease Study, 2015; Diabetol Metab Syndr. 2017; 9: 18.

14. GARCES TS, et al. Tendência de mortalidade por diabetes mellitus. Revista de Enfermagem UFPE online, 2018; 12(12): 3231-8

15. GARCIA C, et al. Estado nutricional e as comorbidades associadas ao Diabetes Mellitus Tipo 2 no idoso. Estudos inderdisciplinares do envelhecimento, 2016; 21(1): 205-216. 
16. GIMENES C, et al. Profile of Hiperdia patients in the municipality of Barra Bonita, Sao Paulo state. Fisioterapia em movimento, 2016; 29(4):731-9

17. GINTER E, SIMKO V. Type 2 diabetes mellitus, pandemic in 21st century. Adv Exp Med Biol, 2012; 771: $42-50$.

18. GLASER N. Clinical features and diagnosis of diabetic ketoacidosis in children and adolescents. UpToDate. 2019. Disponivel em https://www.uptodate.com/contents/diabetic-ketoacidosis-in-children-clinical-features-and-diagnosis>. Acesso em 18 de fevereiro de 2020.

19. GOMES MB, et al. Prevalence of adults with type 1 diabetes who meet the goals of care in daily clinical practice: A Nationwide multicenter study in Brazil. Diabetes Res Clin Pract, 2012; 97(1): 63-70.

20. IDF. International Diabetes Federation. Diabetes Atlas. IDF 9 Edição, 2019. Disponível em: https://www.diabetesatlas.org/en/. Acessado em: 20 de março de 2020.

21. JÚNIOR EVS, et al. Internações, óbitos e custos hospitalares por diabetes mellitus. Revista enfermagem UFPE on line, 2019; 13: e240388

22. LEVITSKY LL, et al. Epidemiology, presentation, and diagnosis of type 1 diabetes mellitus in children and adolescents. UpToDate. 2019. Disponivel em:https://www.uptodate.com/contents/epidemiology-presentation-and-diagnosis-oftype-1-diabetes-mellitus-in-children-and-adolescents. Acesso em 8 de fevereiro de 2020

23. MARQUES ADB, et al. Associação entre internação hospitalar por diabetes mellitus e amputação de pé diabético. Revista electroníca trimestral de enfermeira, 2018; 17: 238-266.

24. MENEZES LCG De, et al. Pesquisa ação: Práticas de autocuidado das pessoas com pé diabético. Revista de Enfermagem UFPE Online, 2017; 11(Supl. 9):3558-66

25. NCD Risk Factor Collaboration (NCD-RisC). Worldwide trends in diabetes since 1980: a pooled analysis of 751 population-based studies with 4.4 million participants. Lancet. 2016 Apr 9; 387(10027): 1513-1530.

26. PIMENTA FB, et al. Fatores associados a doenças crônicas em idosos atendidos pela Estratégia de Saúde da Família. Ciência \& Saúde Coletiva, 2015; 20(8): 2489-2498

27. PRADO MAMB, et al. Diabetes em idosos: uso de medicamentos e risco de interação medicamentosa. Ciência \& Saúde Coletiva, 2016; 21(11): 3447-3458.

28. QUEIROZ PC, et al. Prevalência das complicações micro e macrovasculares e de seus fatores de risco em pacientes com diabetes mellitus e síndrome metabólica. Rev Soc Bras Clin Med, 2011; 9(4): 254-258.

29. SANTOS AL, et al. Assistência às pessoas com Diabetes no HIPERDIA: Potencialidades e limites na perspectiva de enfermeiros. Texto \& Contexto Enfermagem, 2018; 27(1): e2630014.

30. SANTOS AL, et al. Tendência de hospitalizações por diabetes mellitus: implicações para o cuidado em saúde. Acta Paul Enferm, 2015; 28(5): 401-7

31. SANTOS ICRV, et al. Prevalência e fatores associados a amputações por pé diabético. Ciência \& Saúde Coletiva, 2013; 18(10): 3007-3014.

32. SANTOS JC, MOREIRA TMM. Fatores de risco e complicações em hipertensos/diabéticos de uma regional sanitária do nordeste brasileiro. Rev Esc Enferm, 2012; 46(5): 1125-1132.

33. SCHMIDT MI, et al. Doenças crônicas não transmissíveis no Brasil: carga e desafios atuais. The Lancet, 2011; 61-73.

34. STOPA SR, et al. Diabetes autorreferido em idosos: comparação das prevalências e medidas de controle. Revista de Sáude Pública, 2014; 48(4): 554-562.

35. TANG ZQ, et al. Gender Differences of Lower Extremity Amputation Risk in Patients With Diabetic Foot: A MetaAnalysis. Int J Low Extrem Wounds, 2014; 13(3): 197-204.

36. WEINTROB AC, et al. Clinical manifestations, diagnosis, and management of diabetic infections of the lower extremities. UpToDate. 2018. Disponivel em:< https://www.uptodate.com/contents/clinical-manifestations-diagnosisand-management-of-diabetic-infections-of-the-lower-extremities>. Acesso em 10 de fevereiro de 2020

37. WEXLER DJ, et al. Overview of general medical care in nonpregnant adults with diabetes mellitus. UpToDate. 2020. Disponivel em:< https://www.uptodate.com/contents/overview-of-general-medical-care-in-nonpregnant-adults-withdiabetes-mellitus>. Acesso em: 8 de abril de 2020

38. WU T, et al. Metabolomics window into diabetic complications. J Diabetes Investig, 2018; 9: 244- 255

39. ZHANG P, et al. Global epidemiology of diabetic foot ulceration: a systematic review and meta-analysis. Nanjing, China Department of Endocrinology, Nanjing Drum Tower Hospital, 2016. doi: 10.1080/07853890.2016.1231932 